\title{
Emergence of novelties: a thermodynamic approach to ecosystems
}

\author{
E. Tiezzi, R. Ridolfi \& N. Marchettini \\ Department of Chemical and Biosystems Sciences, University of Siena, \\ Italy
}

\begin{abstract}
The First Principle of Thermodynamics is concerned with conservation, the Second Principle with evolution. Time, oscillations, instability and chaos are accorded scientific dignity by the Second Principle.

Energy and matter are conservative properties of the biosphere (First Principle of Thermodynamics). Their organization and the information embodied in the history of energy and matter are evolutionary properties: such properties regard the thermodynamics of far-from-equilibrium systems. At equilibrium, energy and matter are blind; far from equilibrium they begin to see (Ilya Prigogine). This paper shows the emergence of novelties in some particular cases.
\end{abstract}

\section{Introduction}

At all levels we observe events associated with emergence of novelty, that we can in turn associate with the creative power of Nature (Ilya Prigogine).

Prigogine's view introduces the transition from space culture to time culture, ascribing the scientific revolution at the end of the 20th century to the emerging properties, events and narrative elements of biological history. Concepts such as dissipative structures and self organization have become quite popular.

Ecosystems arise and evolve stochastically by co-evolution and selforganization. They are complex systems, the components of which are all interconnected, and they do not obey linear deterministic laws.

As underlined by the palaeontologist Roberto Fondi: 
"Cells differ from other physical systems by virtue of the increased complexity inherent in their epigenetic development, or in other words due to a series of geneses, each of which creates new structures and new functions. No machine or inanimate physical system can increase its complexity as can the simplest living cell”.

In the framework of evolutionary physics, we would deal with goal functions instead of state functions, with configurations of evolutionary processes instead of static molecular structures. The ecodynamic models have to be based on relations evolving in time; "far from equilibrium thermodynamics" (Prigogine) assumes upon itself the role of foundation of a new description of nature.

Far from equilibrium, we witness new states of matter having properties sharply at variance with those of equilibrium states. We must therefore introduce the foundations of irreversibility into our basic description of nature.

The evolutionary process is such that systems become more and more complex and organized. Biological diversity is the product of long-term interactions at a genealogical and ecological level: the genealogical interactions regard the dissipation of entropy by irreversible biological processes; the ecological interactions regard entropy gradients in the environment.

It is a continuous emergence of novelties leading to biodiversities.

Geological, meteorological, ecological, oceanographic and biological studies demonstrate that the life of every organism is part of a large scale process that involves the metabolism of the whole planet. Biological activity is a planetary property, a continuous interaction of atmospheres, oceans, plants, animals, microorganisms, molecules, electrons, energy and matter, all part of the whole. The role of each of these components is essential for the maintenance of life.

Today we know that life is a set of relations and coevolutions reaching us from distant biological eras; an infinite set of interactions between molecules and cells, between atmospheres and living beings, between biological species and ecosystems and we know that life is more characteristic of these systemic interactions than of a single individual. Lucretius was right in saying that life is passed from one being to the other, given in property to no one but in use to all.

On one hand, the use of time-reversible classical and quantum physical chemistry approaches for studying matter at molecular level and the behaviour of simple molecular systems has greatly improved our understanding. On the other hand, we need new approaches and new time-irreversible theories to describe the behaviour of ecosystems.

\section{Discussion}

\subsection{The oscillating reactions}

A typical manifestation of nonlinear behaviour in biology is self-sustained oscillations [1]. Examples can be found at all levels of biological organization, from molecular to supracellular, or even ecosystems, with periods of oscillation ranging from seconds to days or years. The beating of the heart is the most 
obvious of the rhythms that sustain life. Other examples are no less important: Bcells in the pancreas produce insulin in squirts; these periodic pulses are themselves associated with rhythmic oscillations in calcium concentrations. Rhythmic behaviour also arises at metabolic network level: it is a little-known but very interesting fact that hormone levels in the human bloodstream peak five or six times a day, providing an oscillation in key control species for many physiological processes.

Generation of patterns and shapes is a ubiquitous phenomenon in nature, intimately linked with manifestations of oscillatory behaviour. It occurs from microscopic (e.g. self-organization in cells) to macroscopic level (e.g. the mantle of certain animals) in living and non-living systems alike. At every level of complexity, common features seem to relate ecosystem dynamics with the development or organization of biological and chemical systems (the preypredator model shares identical characteristics with chemical oscillators). Understanding pattern generation is thus inherent to understanding the notion of complexity in open systems $[1,2]$.

Stationary (or slowly varying) patterns, that occur in morphogenesis, and time-dependent patterns, which involve propagating waves, are an important example of structures generated by chemical and biochemical systems. The Belousov-Zhabotinsky reaction seems to have both types of patterns, depending on specific conditions $[3,4]$.

The basic mechanism of morphogenesis, namely differentiation of tissues from stem cells, was proposed 50 years ago by Turing [5], who pointed out that if morphogens obey a reaction-diffusion equation, they may undergo symmetrybreaking transitions. This, in turn, generates spatially organized states, now known as Turing structures, which may explain the initial stages of cell aggregation and development. In particular, if we consider a generic reactiondiffusion system

$$
\frac{\partial C_{i}}{\partial t}=D_{i} \nabla^{2} C_{i}+f_{i}\left(\ldots, C_{i}, \ldots, C_{j}, \ldots\right)
$$

where $C_{i}$ is the concentration of the $i$ th species participating in the reaction, $f_{i}$ is the nonlinear kinetics equation describing the reaction and $D_{i}$ is the diffusion coefficient of species $i$. Assuming that eqn (1) has a homogeneous steady state solution, $f_{i}\left(C_{s}\right)=\mathrm{O}$, let us consider the evolution of a small perturbation $c_{s}$ around $C_{s}$ and separate it in Fourier space,

$$
c_{s}=\sum_{k} a_{k} e^{\lambda_{k} t+i \bar{k} \cdot \bar{r}}
$$

where $\lambda_{\kappa}$ is the growth rate of the mode with a wave vector. Substituting eqn (2) in eqn (1) and retaining only the linear term, we obtain an eigenvalue equation for $\lambda_{\kappa}$ for the linear operator 


$$
L_{i j}=F_{i j}-D_{i} k^{2} \delta_{i j}
$$

where $F_{i j}$ is the Jacobian matrix of the kinetic function $f_{i}$. For a certain non-zero mode $k$, Turing instability occurs when the real part of the eigenvalue $\lambda_{\kappa}$ of operator (3) becomes positive, so that the homogeneous steady state becomes unstable and the system undergoes a transition from the homogeneous state to a patterned state. Turing structures are characterized by a constant wavelength and time-independent behaviour. They are generated when the diffusion coefficients of the morphogens (in the simplest case one activator and one inhibitor) are very different.

Target patterns where pulses are emitted periodically from the same leading centre (pacemaker) have been observed in a host of reaction-diffusion systems that generate chemical oscillations. These systems occasionally produce more complex structures, for example outward rotating spirals [6]. The simultaneous presence of propagating waves and stationary patterns was found when the BZ reaction was carried out in the anisotropic environment of a lipid/water binary system. In order to mimic the biological complexity of a cell environment, the Siena Ecodynamics group studied the BZ oscillator in the aqueous compartment of different phospholipid/water lamellar phases [7, 8]. All the lipids studied are major components of plasma membranes. Lipids in water are a good model for biological environments, since different aqueous domains in living systems are usually separated by a membrane with a single phospholipid bilayer as basic structural unit. Moreover, many systems have several bilayers stacked together; examples include myelin structure around nerve axons [9], membranes in retinal rods [10] and the extracellular lipid matrix of the stratum corneum [11]. The results of our work were compared with interesting examples of true biological systems.

Rhythms or oscillations may arise at any level of nature [12]. We usually think of these levels as going from subatomic particles upward: Quarks $\rightarrow$ Atoms $\rightarrow$ Molecules $\rightarrow$ Cells $\rightarrow$ Organisms $\rightarrow$ Societies $\rightarrow$ Ecological Systems $\rightarrow$ Biosphere. This is the traditional reductionist approach to science. Some scientists hope that by breaking the world into smaller and smaller pieces they will eventually achieve an understanding that will enable them to provide a workable description of the whole.

The big question is whether there is a unified theory for the ways in which elements of a system organize themselves to produce behaviours typical of large classes of systems. In complex systems, we accept that processes occurring simultaneously at different scales or levels are important, and the intricate behaviour of the whole system depends on its units in a non-trivial way. Description of the whole system's behaviour requires a qualitatively new theory because the laws governing this behaviour are qualitatively different from those governing the individual units. For example, who could find the law governing the intricate pattern of electrical activity produced by the brain by studying a single neuron? In complex systems randomness and determinism are both 
relevant to the system's overall behaviour. Complex systems exist on the edge of chaos: their behaviour may be almost regular but may change dramatically and stochastically in time and/or space as a result of small changes in conditions.

Returning to the above scheme, it is evident that universal dynamic behaviours (bistability, oscillations, chaos, waves, etc.) can be observed at many levels, including a top planetary level. These phenomena transcend the traditional organizational hierarchy.

\subsection{Emergence of novelties}

The $\mathrm{BZ}$ reaction in presence of lipids gives rise to emergence of novelties. A stationary Turing-like Labyrinthine structure was observed in the BZ/DPPC system with $18 \% \mathrm{w} / \mathrm{w}$ lipid content. Such patterns, once they were formed, were able to persist for about 20 minutes without changing position. At the same concentration we also found that inwardly rotating spirals coexisted with the Turing structures in different regions of the sample.

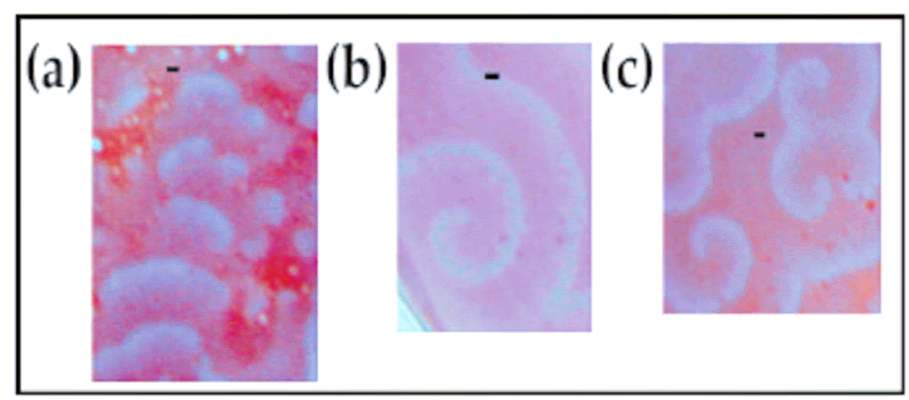

Figure 1: $\quad$ BZ/DPPC system with $25 \% \mathrm{w} / \mathrm{w}$ lipid content, horizontal bar scales $1 \mathrm{~mm}$. (a) striped standing waves. (b) Inwardly rotating spirals. (c) Spiral-like structures, generated from collision between traveling waves and spirals.

When the system reached $25 \% \mathrm{w} / \mathrm{w}$ of lipid content, inwardly rotating spirals and spiral-like structures (fig. 1) coexisted with striped standing waves. In all the systems investigated, waves started at the edge of the reactor, except for few cases in which pacemaker structures appeared in the middle of the dish, and slowly diffused toward the center. This phenomenon was probably favored by the zero-flux boundary conditions. For lipid concentrations higher than $30 \%$ w/w, all systems exhibited only few blue filled circular "spots" and no waves or other interesting structures were observed.

Water up to $70 \%$ seems necessary to give rise to the emergence of novelties.

\section{References}

[1] Murray, J.D., Mathematical Biology, Springer-Verlag, New York, 2002. 
[2] Ball, P., The Self-Made Tapestry: Pattern Formation in Nature, Oxford University Press, Oxford, 1999.

[3] Tyson, J. J. and Fife, P. C., Target patterns in a realistic model of the Belousov-Zhabotinsky reaction, J. Chem. Phys., 73, 2224-2237, 1980.

[4] Vanag, V. K. and Epstein, I. R., Pattern Formation in a Tunable Medium: The Belousov-Zhabotinsky Reaction in an Aerosol OT Microemulsion, Phys. Rev. Lett., 87, 228301-1 - 228301-4, 2001.

[5] Turing, A. M., The Chemical Basis of Morphogenesis, Philos. Trans. Roy. Soc. London B, 327, 37-52, 1952.

[6] R. Kapral and K. Showalter, eds., Chemical Waves and Patterns, Kluwer, Dordrecht (NL), 1995.

[7] Magnani, A., Marchettini, N., Ristori, S., Rossi, C., Rossi, F., Rustici, M., Spalla, O. and Tiezzi, E., Chemical Waves and Pattern Formation in the 1,2-Dipalmitoyl-sn-glycero-3-phosphocholine/Water lamellar system, $J$. Am. Chem. Soc., 126, 11406-11407, 2004.

[8] Marchettini, N., Ristori, S., Rossi, F. and Rustici, M., An Experimental Model for Mimicking Biological Systems: The Belousov-Zhabotinsky Reaction in Lipid Membranes, International Journal of Ecodynamics, 1, 55-63, 2006.

[9] Davis, A. D., Weatherby, T. M., Hartline, D. K. and Lenz, P. H., MyelinLike Sheaths in Copepod Axons, Nature, 398, 571-571, 1999.

[10] Dratz, E. A. and Hargrave, P.A., The Structure of Rhodopsin and the Rod Outer Segment Disk Membrane, Trends Biochem. Sci., 8, 128 -131, 1983.

[11] Sparr, E. and Wennerström, H., Responding Phospholipid Membranes Interplay between Hydration and Permeability, Biophys J., 81, 1014-1028, 2001.

[12] Strogatz, S., SYNC: The Emerging Science of Spontaneous Order, Hyperion books, New York, 2003. 